\title{
Emergent Abelian Gauge Fields from Noncommutative Gravity ${ }^{\star}$
}

\author{
Allen STERN
}

Department of Physics and Astronomy, University of Alabama, Tuscaloosa, Al 35487, USA

E-mail: astern@bama.ua.edu

Received December 30, 2009, in final form February 14, 2010; Published online February 18, 2010

doi:10.3842/SIGMA.2010.019

\begin{abstract}
We construct exact solutions to noncommutative gravity following the formulation of Chamseddine and show that they are in general accompanied by Abelian gauge fields which are first order in the noncommutative scale. This provides a mechanism for generating cosmological electromagnetic fields in an expanding space-time background, and also leads to multipole-like fields surrounding black holes. Exact solutions to noncommutative Einstein-Maxwell theory can give rise to first order corrections to the metric tensor, as well as to the electromagnetic fields. This leads to first order shifts in the horizons of charged black holes.
\end{abstract}

Key words: noncommutative gravity; Groenewold-Moyal star; exact solutions

2010 Mathematics Subject Classification: 83D05; 53D55; 81T75

\section{Introduction}

A number of arguments point to a breakdown of the Riemannian description of space-time at the Planck scale. In particular, it was been argued by Doplicher et al. [1] that a new set of uncertainty relations for distance and time measurements are relevant at the Planck energy scale. These uncertainty relations are naturally realized within the context of noncommutative geometry, where space-time coordinates are replaced by noncommuting operators. This indicates that noncommutative space-time geometry may be the appropriate setting for Planck scale gravity. Among other things, it has the potential of resolving the point singularities which plague general relativity, since the standard notion of 'points' is absent in such a setting ${ }^{1}$.

Corrections to the metric tensor due to noncommutative geometry have been computed for a number of solutions to general relativity $[4,5,6]$. However, so far, the prospects of observing these corrections are not very promising. This is due to the smallness of the noncommutative scale $^{2}$, plus the fact that the previously obtained corrections to the metric tensor are quadratic in the noncommutative scale $[7,8]$. Moreover, the procedure used to obtain the above corrections has some ambiguity. It does not involve solving the noncommutative analogue of Einstein equations, which are not unique. Rather, one maps known solutions of general relativity to the noncommutative theory using the Seiberg-Witten map [9]. A noncommutative analogue of the metric tensor can be defined in order to then give a physical interpretation of the results, but the definition is not unique.

*This paper is a contribution to the Special Issue "Noncommutative Spaces and Fields". The full collection is available at http://www.emis.de/journals/SIGMA/noncommutative.html

${ }^{1}$ This issue was addressed in the simpler context of a noncommutative deformation of electrodynamics where it was shown that noncommutative effects tend to screen the Coulomb singularity at leading order in the deformation $[2,3]$.

${ }^{2}$ Conservative estimates from Hydrogen spectra put limits on the noncommutative scale at around a few $\mathrm{GeV}[2,3]$. 
Here we shall examine exact solutions to noncommutative gravity, and show that they can give rise to effects which are linear in the noncommutative scale when additional gauge fields are present in the theory. Such additional fields are in fact necessary if one follows the approach to noncommutative gravity of Chamseddine [10,11]. The latter is based on the gauge theory formulation of general relativity. Because the relevant gauge group for gravity is non unitary, one needs to enlarge the gauge group in order to pass to the noncommutative theory. This requires adding at least two Abelian gauge fields to the theory. Their coupling to point particles was shown to be identical to that of electromagnetism (although one of them is associated with a noncompact direction of the gauge group) [12]. We shall examine several standard solutions of general relativity, and show that the additional gauge fields pick up first order contributions when going to the noncommutative gravity theory. Starting with the Robertson-Walker metric, this then provides a mechanism for generating cosmological electromagnetic fields. Conversely, if an electromagnetic field is present in a solution to the Einstein-Maxwell equations, it can induce first order corrections in the metric tensor.

Different formulations of noncommutative gravity have been proposed. Among them is the approach of Wess and collaborators [13], where the full diffeomorphism group is preserved. Exact solutions to the latter have been recently discussed in $[14,15]$ which preserve the isometries of the corresponding commutative solution. (See also [16].) Actually, exact solutions can be obtained for a wide class of noncommutative gravity theories. For this one only needs to require a) that the commutative solutions are associated with some isometry group $\mathcal{I}$ and b) that the noncommutative field equations are deformations of the commutative equations obtained in a canonical fashion. Here the term 'canonical' means that pointwise products appearing in the commutative theory get replaced by star products. As discussed below, we shall utilize the Seiberg-Witten map [9] in order to make contact with a theory of space-time. (This differs from $[14,15]$.) Because the Seiberg-Witten map can be problematic for some noncommutative manifolds, we shall specialize to embeddings of the Moyal-Weyl plane, with its realization in terms of the Groenewold-Moyal star product [17, 18]. It is defined in terms of dimensionfull space-time independent parameters $\Theta^{\mu \nu}$, setting the noncommutative scale. One can choose $\Theta^{\mu \nu}$ such that the Groenewold-Moyal star product acts trivially (i.e., as a pointwise product) between any two functions associated with the isometry group $\mathcal{I}$. Noncommutative field equations evaluated for such functions are then equivalent to commutative field equations, and so solutions to commutative gravity with isometry group $\mathcal{I}$ must also be solutions to the noncommutative theory.

The final task is then to give a space-time interpretation to the noncommutative solutions. Unlike in previous treatments, we do not try to define a noncommutative analogue of the metric tensor for this purpose. We instead use the Seiberg-Witten map [9] to map noncommutative solutions onto the space of commutative field configurations, where the standard metric tensor, as well as other familiar quantities of general relativity, can be employed. The Seiberg-Witten map can then potentially generate first order shifts in both the metric tensor and the Abelian gauge fields of the commutative solution. Because $\Theta^{\mu \nu}$ are space-time independent, these shifts will in general break various space-time symmetries associated with the commutative solution. We carry out the procedure for three examples: the flat expanding universe, the Schwarzschild black hole and the Reissner-Nordström black hole. First order Abelian gauge fields emerge in the first two examples, and first order corrections to the metric tensor result in the last example.

The organization of this article is as follows. We review the noncommutative $G L(2, C)$ gauge theory of [11] in Section 2, along with its Seiberg-Witten map from the commutative theory in Section 3. A prescription for finding exact solutions to the noncommutative Einstein equations is described in Section 4. We apply the procedure to the case of the flat expanding universe in Section 5 and the Schwarzschild metric in Section 6. We generalize the procedure in Section 7 in order to find exact solutions to the noncommutative Einstein-Maxwell system and apply it 
to the case of Reissner-Nordström black hole in Section 8. Concluding remarks are made in Section 9. Expressions for the noncommutative gauge variations, the curvature and torsion and the Seiberg-Witten map are given for component one forms in the appendix.

\section{Noncommutative $G L(2, C)$ gauge theory of gravity}

The standard gauge theory formalism for gravity $[19,20,21,22]$ is expressed in terms of vierbeins $e_{\mu}^{a}$ and spin connections $\omega_{\mu}^{a b}=-\omega_{\mu}^{b a} \cdot a, b, \ldots=0,1,2,3$ denote Lorentz indices which are raised and lowered with the flat metric tensor $\eta_{a b}=\operatorname{diag}(-1,1,1,1)$, while $\mu, \nu, \ldots$ are space-time indices which are lowered with the space-time metric

$$
g_{\mu \nu}=e_{\mu}^{a} e_{\nu}^{b} \eta_{a b}
$$

For convenience we use spinor notation of [23] and introduce the matrix-valued one forms

$$
e=e_{\mu}^{a} \gamma_{a} d x^{\mu}, \quad \omega=\frac{1}{2} \omega_{\mu}^{a b} \sigma_{a b} d x^{\mu},
$$

with $\left\{\gamma_{a}, \gamma_{b}\right\}=2 \eta_{a b} 11$ (1l denotes the unit matrix) and $S L(2, C)$ generators $\sigma_{a b}=-\frac{i}{4}\left[\gamma_{a}, \gamma_{b}\right]$. $[$,$] and \{$,$\} denote the matrix commutator and anticommutator, respectively. Infinitesimal$ variations $\delta_{\lambda}$ of $\omega$ and $e$ induced by local $S L(2, C)$ transformations are given by

$$
\delta_{\lambda} \omega=d \lambda+i[\omega, \lambda], \quad \delta_{\lambda} e=i[e, \lambda]
$$

where $\lambda=\frac{1}{2} \lambda^{a b} \sigma_{a b}$ is infinitesimal. Such transformations leave the metric tensor (2.1) invariant. The spin curvature $R$ and torsion $T$ are constructed from $\omega$ and $e$ according to

$$
\begin{aligned}
& R=\frac{1}{2} R_{\mu \nu}^{a b} \sigma_{a b} d x^{\mu} \wedge d x^{\nu}=d \omega+i \omega \wedge \omega, \\
& T=T_{\mu \nu}^{a} \gamma_{a} d x^{\mu} \wedge d x^{\nu}=d e+i(\omega \wedge e+e \wedge \omega),
\end{aligned}
$$

the latter of which is set to zero in the usual metric description of general relativity. $R$ and $T$ satisfy the Bianchi identities

$$
\begin{aligned}
& d R=i(R \wedge \omega-\omega \wedge R), \\
& d T=i(R \wedge e-e \wedge R+T \wedge \omega-\omega \wedge T) .
\end{aligned}
$$

The canonical procedure to go from a commutative to a noncommutative theory is to replace the pointwise products of the commutative theory by star products, more specifically Groenewold-Moyal star products [17, 18], given by

$$
\star=\exp \left\{\frac{i}{2} \Theta^{\mu \nu} \overleftarrow{\partial_{\mu}} \overrightarrow{\partial_{\nu}}\right\}
$$

where $\Theta^{\mu \nu}=-\Theta^{\nu \mu}$ are constant matrix elements denoting the noncommutativity parameters and $\overleftarrow{\partial_{\mu}}$ and $\overrightarrow{\partial_{\mu}}$ are left and right derivatives, respectively, with respect to some space-time coordinates $x^{\mu}$. If we call $\hat{A}, \hat{B}, \ldots$ the noncommutative analogues of the matrix-valued forms $A, B, \ldots$ of the commutative theory, then the matrix-valued star commutator $[\hat{A}, \hat{B}]_{\star}=\hat{A} \star$ $\hat{B}-\hat{B} \star \hat{A}$ replaces the matrix commutator $[A, B]$ when we go to the noncommutative theory. Gauge theories based on non unitary groups, such as $S L(2, C)$, do not have a straightforward generalization to noncommutative gauge theories. (See [24, 25].) For this note that the $S L(2, C)$ algebra is not realized with the star commutator. This follows because the star commutator of matrix valued forms can be decomposed in terms of commutators, as well as anticommutators, of the algebra generators. The star commutators of forms proportional to $\sigma_{a b}$ and $\gamma_{a}$ then generate 
the entire Clifford algebra. Such star commutators will appear upon trying to lift the gauge variations in (2.2) to noncommutative variations. The commutators $[\omega, \lambda]$ and $[e, \lambda]$ in $(2.2)$ get replaced by

$$
\begin{aligned}
& {[\hat{\omega}, \hat{\lambda}]_{\star}=\frac{1}{8}\left\{\hat{\omega}^{a b}, \hat{\lambda}^{c d}\right\}_{\star}\left[\sigma_{a b}, \sigma_{c d}\right]+\frac{1}{8}\left[\hat{\omega}^{a b}, \hat{\lambda}^{c d}\right]_{\star}\left\{\sigma_{a b}, \sigma_{c d}\right\},} \\
& {[\hat{e}, \hat{\lambda}]_{\star}=\frac{1}{4}\left\{\hat{e}^{a}, \hat{\lambda}^{b c}\right\}_{\star}\left[\gamma_{a}, \sigma_{b c}\right]+\frac{1}{4}\left[\hat{e}^{a}, \hat{\lambda}^{b c}\right]_{\star}\left\{\gamma_{a}, \sigma_{b c}\right\},}
\end{aligned}
$$

where $\hat{\omega}=\frac{1}{2} \hat{\omega}_{\mu}^{a b} \sigma_{a b} d x^{\mu}, \hat{e}=\hat{e}_{\mu}^{a} \gamma_{a} d x^{\mu}$ and $\hat{\lambda}=\frac{1}{2} \hat{\lambda}^{a b} \sigma_{a b}$ are the noncommutative analogues of the connection one form $\omega$, the vierbein one form $e$ and the gauge parameters $\lambda$, respectively, and $\{,\}_{\star}$ denotes the star anticommutator, $\{\hat{\alpha}, \hat{\beta}\}_{\star}=\hat{\alpha} \star \hat{\beta}+\hat{\beta} \star \hat{\alpha}$. Using the GroenewoldMoyal star, $\{\hat{\alpha}, \hat{\beta}\}_{\star}$ is real for any two real-valued functions $\hat{\alpha}$ and $\hat{\beta}$, while $[\hat{\alpha}, \hat{\beta}]_{\star}$ is imaginary. The anticommutator $\left\{\sigma_{a b}, \sigma_{c d}\right\}$ appearing in (2.4) is a linear combination of $\gamma_{5}$ and the unit matrix 11. Closure therefore requires that we enlarge the gauge group to $G L(2, C)$ in order to include these two generators. Following [11] one then introduces $G L(2, C)$ connections $\hat{\mathcal{A}}$ and gauge parameters $\hat{\Lambda}$ which can be decomposed as

$$
\hat{\mathcal{A}}=\hat{\omega}+\hat{A}^{(1)} 11+i \hat{A}^{(2)} \gamma_{5}, \quad \hat{\Lambda}=\hat{\lambda}+\hat{\alpha}^{(1)} 11+i \hat{\alpha}^{(2)} \gamma_{5},
$$

where $\hat{A}^{(S)}, S=1,2$, are Abelian connection one forms, $\hat{A}^{(S)}=\hat{A}_{\mu}^{(S)} d x^{\mu}$, and $\hat{\alpha}^{(S)}$ are functions on space-time. The anticommutator $\left\{\sigma_{a b}, \gamma_{c}\right\}$ appearing in (2.5) is a linear combination of $\gamma_{5} \gamma_{c}$, and so closure requires that one generalizes the vierbein one forms $\hat{e}$ to

$$
\hat{\mathcal{E}}=\hat{e}+\hat{f}, \quad \hat{f}=\hat{f}_{\mu}^{a} \gamma_{5} \gamma_{a} d x^{\mu} .
$$

A consistent set of noncommutative $G L(2, C)$ gauge variations can then be defined according to

$$
\delta_{\hat{\Lambda}} \hat{\mathcal{A}}=d \hat{\Lambda}+i[\hat{\mathcal{A}}, \hat{\Lambda}]_{\star}, \quad \delta_{\hat{\Lambda}} \hat{\mathcal{E}}=i[\hat{\mathcal{E}}, \hat{\Lambda}]_{\star}
$$

These variations are decomposed in terms of component fields $\hat{\omega}^{a b}, \hat{A}^{(S)}, \hat{e}^{a}$ and $\hat{f}^{a}$ in (A.1) in the appendix. The noncommutative $G L(2, C)$ curvature $\hat{\mathcal{F}}$ and torsion $\hat{\mathcal{T}}$ are given by

$$
\begin{aligned}
& \hat{\mathcal{F}}=d \hat{\mathcal{A}}+i \hat{\mathcal{A}} \wedge^{\star} \hat{\mathcal{A}}=\frac{1}{2} \hat{R}^{a b} \sigma_{a b}+\hat{F}^{(1)} 11+i \hat{F}^{(2)} \gamma_{5}, \\
& \hat{\mathcal{T}}=d \hat{\mathcal{E}}+i\left(\hat{\mathcal{A}}{ }^{\star} \hat{\mathcal{E}}+\hat{\mathcal{E}}{ }^{\star} \hat{\mathcal{A}}\right)=\hat{T}^{a} \gamma_{a}+\hat{U}^{a} \gamma_{5} \gamma_{a},
\end{aligned}
$$

where $\wedge^{\star}$ denotes an exterior product where the usual pointwise product between components of the forms replaced by the Groenewold-Moyal star product. The components $\hat{R}^{a b}, \hat{F}^{(S)}, \hat{T}^{a}$ and $\hat{U}^{a}$ of the noncommutative curvature and torsion two forms are given in (A.2) in the appendix. The Bianchi identities now read

$$
\begin{aligned}
& d \hat{\mathcal{F}}=i\left(\hat{\mathcal{F}} \wedge^{\star} \hat{\mathcal{A}}-\hat{\mathcal{A}} \wedge^{\star} \hat{\mathcal{F}}\right), \\
& d \hat{\mathcal{T}}=i\left(\hat{\mathcal{F}} \wedge^{\star} \hat{\mathcal{E}}-\hat{\mathcal{E}} \wedge^{\star} \hat{\mathcal{F}}+\hat{\mathcal{T}} \wedge^{\star} \hat{\mathcal{A}}-\hat{\mathcal{A}} \wedge^{\star} \hat{\mathcal{T}}\right) .
\end{aligned}
$$

\section{First order Seiberg-Witten map}

The Seiberg-Witten map [9] can be used to map the noncommutative gravity theory back to a commutative gravity theory, where quantities like the metric tensor can be straightforwardly defined. However, the commutative gravity theory here is not the standard $S L(2, C)$ gauge theory description of gravity, but rather it is a $G L(2, C)$ gauge theory. The latter is defined in terms of the one forms $\mathcal{A}=\mathcal{A}_{\mu} d x^{\mu}=\omega+A^{(1)} 11+i A^{(2)} \gamma_{5}$ and $\mathcal{E}=\mathcal{E}_{\mu} d x^{\mu}=e^{a} \gamma_{a}+f^{a} \gamma_{5} \gamma_{a}$ 
which are the commutative analogues of $\hat{\mathcal{A}}$ and $\hat{\mathcal{E}}$, respectively. The commutative $G L(2, C)$ gauge variations are given by

$$
\delta_{\Lambda} \mathcal{A}=d \Lambda+i[\mathcal{A}, \Lambda], \quad \delta_{\Lambda} \mathcal{E}=i[\mathcal{E}, \Lambda]
$$

with infinitesimal parameters $\Lambda=\frac{1}{2} \lambda^{a b} \sigma_{a b}+\alpha^{(1)} 11+i \alpha^{(2)} \gamma_{5}$. In terms of component fields $\omega^{a b}$, $A^{(S)}, e^{a}$ and $f^{a}$, this means

$$
\begin{aligned}
& \delta_{\Lambda} \omega^{a b}=d \lambda^{a b}+\omega^{a c} \lambda_{c}{ }^{b}-\omega^{b c} \lambda_{c}{ }^{a}, \\
& \delta_{\Lambda} A^{(S)}=d \alpha^{(S)}, \quad S=1,2, \\
& \delta_{\Lambda} e^{a}=e^{b} \lambda_{b}{ }^{a}+2 f^{a} \alpha^{(2)}, \\
& \delta_{\Lambda} f^{a}=f^{b} \lambda_{b}{ }^{a}+2 e^{a} \alpha^{(2)} .
\end{aligned}
$$

Equation (2.1) is not suitable as a metric tensor for this theory since it is not invariant under the full set of $G L(2, C)$ transformations. The following $G L(2, C)$ invariant metric tensor was given in [12]:

$$
\mathrm{g}_{\mu \nu}=\frac{1}{4} \operatorname{tr} \mathcal{E}_{\mu} \mathcal{E}_{\nu}=e_{\mu}^{a} e_{a \nu}-f_{\mu}^{a} f_{a \nu} .
$$

Because this gravity theory possesses two vierbein fields $e^{a}$ and $f^{a}$, one can construct two sets of torsion two-forms

$$
T^{a}=d e^{a}+\frac{1}{2}\left[\omega^{a b}, e_{b}\right], \quad U^{a}=d f^{a}+\frac{1}{2}\left[\omega^{a b}, f_{b}\right],
$$

which are the commutative analogues of $\hat{T}^{a}$ and $\hat{U}^{a}$.

The Seiberg-Witten map relates the noncommutative degrees of freedom in $\hat{\mathcal{A}}_{\mu}$ and $\hat{\mathcal{E}}_{\mu}$, along with transformation parameters $\hat{\Lambda}$, to their commutative counterparts $\mathcal{A}_{\mu}, \mathcal{E}_{\mu}$ and $\Lambda$, and is defined such that gauge transformations in the commutative theory induce gauge transformations in the corresponding noncommutative theory. Up to first order in $\Theta^{\mu \nu}$ it can be given by

$$
\begin{aligned}
& \hat{\mathcal{A}}_{\mu}=\mathcal{A}_{\mu}+\frac{1}{4} \Theta^{\rho \sigma}\left\{\mathcal{A}_{\rho}, \partial_{\sigma} \mathcal{A}_{\mu}+\mathcal{F}_{\sigma \mu}\right\}+\mathcal{O}\left(\Theta^{2}\right), \\
& \hat{\mathcal{E}}_{\mu}=\mathcal{E}_{\mu}+\frac{1}{2} \Theta^{\rho \sigma}\left\{\mathcal{A}_{\rho}, \partial_{\sigma} \mathcal{E}_{\mu}+\frac{i}{2}\left[\mathcal{A}_{\sigma}, \mathcal{E}_{\mu}\right]\right\}+\mathcal{O}\left(\Theta^{2}\right), \\
& \hat{\Lambda}=\Lambda+\frac{1}{4} \Theta^{\rho \sigma}\left\{\mathcal{A}_{\rho}, \partial_{\sigma} \Lambda\right\}+\mathcal{O}\left(\Theta^{2}\right)
\end{aligned}
$$

The resulting first order expressions for the component one forms $\hat{\omega}^{a b}, \hat{A}^{(S)}, \hat{e}^{a}$ and $\hat{f}^{a}$ are given in (A.3) of the appendix ${ }^{3}$.

\section{Exact solutions to noncommutative gravity}

Here we do not specify any particular dynamics for noncommutative gravity, but only demand that the field equations are deformations of the standard Einstein equations, with deformation parameters $\Theta^{\mu \nu}$, and are obtained in the canonical way, i.e., by replacing pointwise products with Groenewold-Moyal star products. Such a procedure is, of course, not unique. One can nevertheless write down exact solutions for the noncommutative theory, as we describe below.

Say a solution to (commutative) gravity is associated with some nontrivial isometry group $\mathcal{I}$, which is generated by $n$ Killing vectors $K_{\alpha}, \alpha=1, \ldots, n$. Denote by $V_{A}, A=1, \ldots, 4-n$, the remaining independent vectors normal to $K_{\alpha}$. Now define $\Theta^{\mu \nu}$ such that

$$
\Theta^{A B} V_{A} \otimes V_{B}=0
$$

\footnotetext{
${ }^{3}$ For a related Seiberg-Witten map, see [26].
} 
If all $K_{\alpha}$ vanishes on a set of functions $\mathcal{F}$, then the bivectors $\Theta^{\alpha B} K_{\alpha} \otimes V_{B}$ and $\Theta^{\alpha \beta} K_{\alpha} \otimes K_{\beta}$ vanish on $\mathcal{F} \otimes \mathcal{F}$. Moreover, from (4.1) it follows that $\Theta^{\mu \nu} \partial_{\mu} \otimes \partial_{\nu}$ vanishes on $\mathcal{F} \otimes \mathcal{F}$. From the definition of the Groenewold-Moyal star (2.3), one gets that the star product of any pair of functions in $\mathcal{F}$ is identical to its corresponding pointwise product. So when evaluated on elements of $\mathcal{F}$, any noncommutative field equation, obtained using the canonical procedure, reduces to the corresponding commutative field equation. Therefore solutions to commutative gravity with isometry group $\mathcal{I}$ must also be solutions to the noncommutative theory. The former solutions are specified by $S L(2, C)$ vierbein and spin connection one forms, which we denote respectively by $e_{(0)}^{a}=e_{(0) \mu}^{a} d x^{\mu}$ and $\omega_{(0)}^{a}=\omega_{(0) \mu}^{a} d x^{\mu}$. Then the corresponding noncommutative solution is given by

$$
\hat{e}_{\mu}^{a}=e_{(0) \mu}^{a}, \quad \hat{\omega}_{\mu}^{a b}=\omega_{(0) \mu}^{a b}, \quad \hat{A}_{\mu}^{(S)}=\hat{f}_{\mu}^{a}=0 .
$$

The above procedure can be adapted for obtaining exact solutions can be applied to a wide class of noncommutative theories, i.e., those obtained in the canonical way. Exact solutions were found in $[14,15]$ to the noncommutative gravity theory of [13]. They were also used to find exact solutions to noncommutative electrodynamics [3].

In order to give a physical interpretation to the exact noncommutative solution (4.2), one can use (3.4) to map the solution to the commutative theory. The $S L(2, C)$ vierbeins $e_{(0) \mu}^{a}$ and spin connections $\omega_{(0) \mu}^{a b}$ pick up no first order corrections from the Seiberg-Witten map. On the other hand, the map does generate nonvanishing first order results for $A_{\mu}^{(S)}$ and $f_{\mu}^{a}$ :

$$
\begin{aligned}
& A_{\mu}^{(1)}=-\frac{1}{16} \Theta^{\rho \sigma}\left[\omega_{(0) a b}\right]_{\rho}\left(\partial_{\sigma} \omega_{(0) \mu}^{a b}+R_{(0) \sigma \mu}^{a b}\right)+\mathcal{O}\left(\Theta^{2}\right), \\
& A_{\mu}^{(2)}=\frac{1}{32} \Theta^{\rho \sigma} \epsilon_{a b c d} \omega_{(0) \rho}^{a b}\left(\partial_{\sigma} \omega_{(0) \mu}^{c d}+R_{(0) \sigma \mu}^{c d}\right)+\mathcal{O}\left(\Theta^{2}\right), \\
& f_{\mu}^{a}=\frac{1}{4} \Theta^{\rho \sigma} \epsilon_{b c d}^{a} \omega_{(0) \rho}^{b c}\left(\partial_{\sigma} e_{(0) \mu}^{d}+\frac{1}{2} \omega_{(0) \sigma}^{d g} e_{(0) g \mu}\right)+\mathcal{O}\left(\Theta^{2}\right),
\end{aligned}
$$

where $R_{(0)}$ is the Lorentz curvature for the solution. Since the $G L(2, C)$ invariant metric tensor (3.2) is quadratic in $f_{\mu}^{a}$, it receives no first order corrections. This is consistent with the usual result that noncommutative gravity corrections to the space-time metric tensor are second order in the noncommutativity parameter $[7,8]$. This, however, would not be the case for solutions of the Einstein-Maxwell equations which are associated with a nonzero Abelian curvature, since then $\hat{A}^{(S)}$ is nonzero. Therefore an Abelian gauge field can generate first order corrections to the metric tensor. We show this in Section 7 and give the example of the Reissner-Nordström black hole solution in Section 8. The induced $f_{\mu}^{a}$ in (4.3) can lead to a first order $G L(2, C)$ torsion $U^{a}=d f^{a}+\mathcal{O}\left(\Theta^{2}\right)$, but its physical meaning is not immediately evident.

In the next two sections we carry out the above procedure to find exact solutions of the noncommutative theory for the case of the flat expanding universe and the Schwarzschild black hole and we obtain the leading order induced Abelian gauge fields in these two examples. For the former example, any choice for the constants $\Theta^{\mu \nu}$ satisfies (4.1) when expressed in terms of comoving coordinates, while for static solutions, (4.1) is always satisfied for the case of spacetime noncommutativity. In addition to (4.1), there is the requirement that the generators of the star product algebra are self-adjoint operators. This is the case for all the examples which follow.

\section{$5 \quad$ Flat expanding universe}

The invariant measure for a flat expanding universe is

$$
d s_{(0)}^{2}=-d t^{2}+a(t)^{2} d x_{i} d x_{i}, \quad i=1,2,3,
$$


where $x_{i}$ span $R^{3}$ and define comoving coordinates. Equation (5.1) gives a solution to the Einstein equations with stress-energy tensor of the form

$$
\mathrm{T}_{\mu \nu}^{S E}=\operatorname{diag}\left(\rho(t), a(t)^{2} p(t), a(t)^{2} p(t), a(t)^{2} p(t)\right),
$$

where $\rho(t)$ and $p(t)$ are the proper energy density and pressure, respectively, and are related to $a(t)$. Vierbein and spin connection one forms consistent with the zero torsion condition of general relativity can be given by

$$
\begin{aligned}
& e_{(0)}^{0}=d t, \quad e_{(0)}^{i}=a(t) d x_{i}, \\
& \omega_{(0)}^{0 i}=\dot{a}(t) d x_{i}, \quad \omega_{(0)}^{i j}=0 .
\end{aligned}
$$

Here all spatial directions correspond to Killing vectors $K_{i}=\frac{\partial}{\partial x^{i}}$. Since only one independent vector $V_{0}=\frac{\partial}{\partial t}$ remains, the condition (4.1) is satisfied for any choice of $\Theta^{\mu \nu}$. Therefore, (4.2) is an exact solution to the noncommutative gravity equations (obtained using the canonical procedure) with stress-energy tensor (5.2), for any choice of $\Theta^{\mu \nu}$. We can thus choose spacespace noncommutativity

$$
\left[x_{i}, x_{j}\right]_{\star}=\Theta^{i j},
$$

or time-space noncommutativity

$$
\left[t, x_{i}\right]_{\star}=\Theta^{0 i},
$$

or both. (5.3) and (5.4) break the three dimensional rotation symmetry. This symmetry breaking will not appear at first order in the metric tensor, but does appear in the electric field at first order. As was remarked previously [15], these commutation relations apply for the comoving coordinates, while the commutators between 'physical' spatial coordinates $y_{i}$ involve the scale factor, $y_{i}=a(t) x_{i}$. As a result, the 'physical' noncommutative scale will become extremely small at earlier scales, and one would expect that this prevents the noncommutative effects from becoming too large at earlier times. This could be desirable, since such effects break rotational invariance. Despite this, as we argue below, the fields might become significant during the inflation era.

Next we substitute into (4.3). A nonvanishing first order potential $A_{\mu}^{(1)}$ only arises for the case of time-space noncommutativity $(5.4)$

$$
A_{0}^{(1)}=\mathcal{O}\left(\Theta^{2}\right), \quad A_{i}^{(1)}=-\frac{1}{4} \Theta^{0 i} \dot{a}(t) \ddot{a}(t)+\mathcal{O}\left(\Theta^{2}\right),
$$

leading to a uniform electric field at first order, directed along $\Theta^{0 i}$,

$$
E_{i}^{(1)}=-\frac{1}{4} \Theta^{0 i}\left(\ddot{a}(t)^{2}+\dot{a}(t) a^{(3)}(t)\right)+\mathcal{O}\left(\Theta^{2}\right) .
$$

A nonvanishing first order potential $A_{\mu}^{(2)}$ only arises for the case of space-space noncommutativity (5.3)

$$
A_{0}^{(2)}=\mathcal{O}\left(\Theta^{2}\right), \quad A_{i}^{(2)}=\frac{1}{8} \epsilon_{i j k} \Theta^{j k} \dot{a}(t)^{3}+\mathcal{O}\left(\Theta^{2}\right),
$$

leading to a uniform electric field at first order, in the $\epsilon_{i j k} \Theta^{j k}$ direction,

$$
E_{i}^{(2)}=\frac{3}{8} \epsilon_{i j k} \Theta^{j k} \dot{a}(t)^{2} \ddot{a}(t)+\mathcal{O}\left(\Theta^{2}\right) .
$$

Since the associated magnetic fields vanish, it follows that the standard Maxwell equations in vacuum are not satisfied. Rather, the dynamics for the Abelian gauge fields is governed by 
a deformed Maxwell action, and as a result, magnetic fields are shielded by the noncommutative vacuum.

If one applies the results (5.5) and (5.6) to the universe at the current time one gets incredibly tiny electric fields, since they go like powers of the Hubble parameter (not to mention the small noncommutative scale $\Theta$ ). The fields grow when we evolve back in time. Yet, they remain insignificant even if we go all the way back to the beginning of the radiation dominated era. For the radiation dominated era we can apply

$$
a(t)=a_{r m}\left(\frac{t}{t_{r m}}\right)^{1 / 2}
$$

where $a_{r m} \sim 3 \times 10^{-4}$ and $t_{r m} \sim 5 \times 10^{4} \mathrm{yr}$, respectively, are the scale factor and time of the radiation-matter equality. Substituting into (5.5) and (5.6) gives

$$
E_{i}^{(1)}=-\frac{1}{16} \Theta^{0 i} \frac{a_{r m}^{2}}{t_{r m} t^{3}}+\mathcal{O}\left(\Theta^{2}\right), \quad E_{i}^{(2)}=-\frac{3}{128} \epsilon_{i j k} \Theta^{j k} \frac{a_{r m}^{3}}{t_{r m}^{3 / 2} t^{5 / 2}}+\mathcal{O}\left(\Theta^{2}\right) .
$$

If we take known upper limits $[2,3]$ for the time-space and space-space noncommutativity scale of $\Theta \lesssim \mathrm{GeV}^{-2}$, and set $t=t_{r m}$, we get $\left|E^{(1)}\right| \lesssim\left(10^{-67} \mathrm{eV}\right)^{2}$ and $\left|E^{(2)}\right| \lesssim\left(10^{-69} \mathrm{eV}\right)^{2}$. These scales are nowhere near those claimed needed for the primordial (magnetic) field $\left(10^{-15}-10^{-25} \mathrm{eV}^{2}\right)$ to seed an amplification process which can then produce the currently observed intergalactic magnetic fields $[27]^{4}$. If we assume that (5.7) is valid all the way back to, say $t \sim 10^{-30} \mathrm{sec}$ (and we take the previous limit for $\Theta$ ), we get $\left|E^{(1)}\right| \lesssim\left(10^{-3} \mathrm{eV}\right)^{2}$ and $\left|E^{(2)}\right| \lesssim\left(10^{-16} \mathrm{eV}\right)^{2}$. These energy scales are still far below the radiation energy $\left(\sim 10^{6} \mathrm{GeV}\right)$ at the time $t \sim 10^{-30}$ sec, and so the fields are not expected to play a role.

On the other hand, the fields $\left|E^{(1)}\right|$ and $\left|E^{(2)}\right|$ can be very large during a prior exponential inflationary era. (Note that the fields depend on derivatives of $a(t)$, which are not continuous across the transition from the inflationary era to the radiation era if one assumes that the exponential inflation was suddenly switched off. The fields therefore undergo a discontinuous jump across the transition.) Upon taking $a(t) \propto e^{H t}$, both $\left|E^{(1)}\right|$ and $\left|E^{(2)}\right|$ go like $H^{4}$ times the noncommutative scale. Since here $H$ is associated with a large energy scale, these fields could be significant during inflation.

\section{Schwarzschild solution}

The Schwarzschild invariant measure may be written as

$$
d s_{(0)}^{2}=\left(-1+\alpha(r)^{2}\right) d t^{2}+\left(\frac{\alpha(r)^{2}}{1-\alpha(r)^{2}} \hat{x}_{i} \hat{x}_{j}+\delta_{i j}\right) d x_{i} d x_{j}, \quad \alpha(r)^{2}=\frac{r_{s}}{r},
$$

where the spatial coordinates $x_{i}$ again span $R^{3} \cdot r=\sqrt{x_{i} x_{i}}$ is the radial coordinate, $\hat{x}_{i}=x_{i} / r$ are unit vectors and $r_{s}=2 G M$ is the Schwarzschild radius. A consistent set of vierbein one forms $e_{(0)}$ is

$$
e_{(0)}^{0}=d t+\frac{\alpha(r) d r}{1-\alpha(r)^{2}}, \quad e_{(0)}^{i}=\alpha(r) \hat{x}_{i} e_{(0)}^{0}+d x_{i} .
$$

The torsion vanishes with the following assignment for the spin connections

$$
\omega_{(0)}^{i 0}=d\left(\alpha(r) \hat{x}_{i}\right)+\alpha(r) \alpha^{\prime}(r) \hat{x}_{i} e_{(0)}^{0}, \quad \omega_{(0)}^{i j}=0,
$$

\footnotetext{
${ }^{4}$ The application of noncommutative physics to the primordial magnetic field problem was suggested earlier in [28], but the field there originates from the matter content of the universe, and is not generated from the Robertson-Walker background, as is the case here.
} 
where the prime denotes differentiation in $r$. Equations (6.1) and (6.2) are valid both inside and outside the event horizon and lead to zero torsion and zero curvature (for $r>0$ ).

As with all static solutions, the Schwarzschild solution has a time-like Killing vector $K_{0}=\frac{\partial}{\partial t}$. Now we identify the spatially directed vectors with $V_{i}=\frac{\partial}{\partial x^{i}}$. From (4.1), we get an exact solution of the noncommutative gravity equations when all space-space components of $\Theta^{\mu \nu}$ vanish. So here we only consider time-space noncommutativity (5.4). The Groenewold-Moyal star product when acting between $t$-independent functions reduces to the pointwise product, and so if the noncommutative gravity equations are obtained in the canonical way, they are equivalent to the commutative gravity equations when evaluated for static fields. Equation (4.2) is then an exact solution of the noncommutative field equations with time-space noncommutativity.

Now substitute into (4.3) to obtain the induced commutative Abelian gauge potentials $A_{\mu}^{(1)}$ and $A_{\mu}^{(2)}$ at leading order. Here we find that the former leads to an electrostatic field and the latter leads to a magnetostatic field originating from the black hole. The vector potential $A_{i}^{(1)}$ is a pure gauge at first order and hence there is no associated first order magnetic field $B_{i}^{(1)}$, while

$$
A_{0}^{(1)}=-\frac{\Theta^{0 i} r_{s}^{2} \hat{x}_{i}}{8 r^{5}}+\mathcal{O}\left(\Theta^{2}\right)
$$

resulting in the electrostatic field

$$
E_{i}^{(1)}=\frac{\Theta^{0 j} r_{s}^{2}}{8} \frac{\delta_{i j}-6 \hat{x}_{i} \hat{x}_{j}}{r^{6}}+\mathcal{O}\left(\Theta^{2}\right)
$$

On the other hand, $A_{0}^{(2)}=\mathcal{O}\left(\Theta^{2}\right)$ and hence there is no associated first order electric field $E_{i}^{(2)}$, while

$$
A_{i}^{(2)}=\frac{\Theta^{0 k} r_{s}^{2}}{8 r^{5}} \epsilon_{i j k} \hat{x}_{j}+\mathcal{O}\left(\Theta^{2}\right)
$$

resulting in the magnetostatic field

$$
B_{i}^{(2)}=\frac{\Theta^{0 j} r_{s}^{2}}{4} \frac{2 \delta_{i j}-3 \hat{x}_{i} \hat{x}_{j}}{r^{6}}+\mathcal{O}\left(\Theta^{2}\right)
$$

The results (6.3) and (6.4) break the rotational symmetry of the Schwarzschild solution and resemble higher multipole fields, with $\Theta^{0 i} r_{s}^{2}$ playing a role analogous to that of a multipole moment. As the fields fall off as $1 / r^{6}$, they are very weak far from the black hole. At the Schwarzschild radius $r=r_{s}$, they both go like $\sim \Theta^{0 i} / r_{s}^{4}$. Since $\Theta^{0 i}$ is constant in this theory, the moments of multiple black holes are aligned, which may lead to a peculiar signature for microscopic black holes.

\section{Exact solutions to noncommutative Einstein-Maxwell theory}

It is straightforward to generalize the procedure of Section 4 to find exact solutions to the combined noncommutative Einstein-Maxwell system. As before, we do not specify any particular dynamics for the noncommutative theory, but only demand that the field equations are deformations of the standard Einstein-Maxwell equations, obtained in the canonical way. In the examples in Sections 5 and 6, solutions to the noncommutative Einstein equations generated Abelian gauge fields which were first order in $\Theta$. Conversely, here we show that solutions to noncommutative Maxwell equations can generate first order corrections to the metric tensor. For this we shall require, as before, that (4.1) is satisfied, and that the generators of the star product algebra are self-adjoint operators. 
Solutions to the commutative Einstein-Maxwell equations are specified by $S L(2, C)$ vierbein and spin connection one forms, $e_{(0)}^{a}$ and $\omega_{(0)}^{a b}$, along with an Abelian potential one form $A_{(0)}=$ $A_{(0) \mu} d x^{\mu}$. Since $G L(2, C)$ gauge theory contains two Abelian gauge fields, we can construct two different exact solutions to noncommutative Einstein-Maxwell theory. Below we first assume that $\hat{A}^{(1)}$ satisfies the noncommutative Maxwell equation and hence identify $A_{(0)}$ with $\hat{A}^{(1)}$, and then we consider the case for $\hat{A}^{(2)}$.

1. $\hat{A}^{(1)}$ satisfies the noncommutative Maxwell equation. If (4.1) holds we have the exact noncommutative solution

$$
\hat{e}_{\mu}^{a}=e_{(0) \mu}^{a}, \quad \hat{\omega}_{\mu}^{a b}=\omega_{(0) \mu}^{a b}, \quad \hat{A}_{\mu}^{(1)}=A_{(0) \mu}, \quad A_{\mu}^{(2)}=\hat{f}_{\mu}^{a}=0 .
$$

Upon applying the Seiberg-Witten map back to the commutative theory, one now can pick up first order corrections to all of the $G L(2, C)$ fields. For the two vierbein fields $e_{\mu}^{a}$ and $f_{\mu}^{a}$, one has

$$
\begin{aligned}
e_{\mu}^{a} & =e_{(0) \mu}^{a}-\Theta^{\rho \sigma} A_{(0) \rho}\left(\partial_{\sigma} e_{(0) \mu}^{a}+\frac{1}{2} \omega_{(0) \sigma}^{a g} e_{(0) g \mu}\right)+\mathcal{O}\left(\Theta^{2}\right), \\
f_{\mu}^{a} & =\frac{1}{4} \Theta^{\rho \sigma} \epsilon_{b c d}^{a} \omega_{(0) \rho}^{b c}\left(\partial_{\sigma} e_{(0) \mu}^{d}+\frac{1}{2} \omega_{(0) \sigma}^{d g} e_{(0) g \mu}\right)+\mathcal{O}\left(\Theta^{2}\right) .
\end{aligned}
$$

The former can then give rise to first order corrections to the classical metric tensor $g_{(0) \mu \nu}$. After substituting the expression for $e_{\mu}^{a}$ into (3.2), one gets the simple result that

$$
\mathrm{g}_{\mu \nu}(x)=g_{(0) \mu \nu}(x)-\Theta^{\rho \sigma} A_{(0) \rho}(x) \partial_{\sigma} g_{(0) \mu \nu}+\mathcal{O}\left(\Theta^{2}\right)=g_{(0) \mu \nu}\left(x+\Theta A_{(0)}\right)+\mathcal{O}\left(\Theta^{2}\right) .
$$

Since $\mathrm{g}_{\mu \nu}$ transforms under diffeomorphisms as a rank 2 tensor, the first order correction cannot be removed by the coordinate redefinition $x \rightarrow x-\Theta A_{(0)}$. From (4.1), the bivector $\Theta^{\mu \nu} \partial_{\mu} \otimes \partial_{\nu}$ gets contributions from $\Theta^{\alpha B} K_{\alpha} \otimes V_{B}$ and $\Theta^{\alpha \beta} K_{\alpha} \otimes K_{\beta}$. However, only the former produces first order corrections to $g_{(0) \mu \nu}$, and from (7.2), they are of the form $\delta g_{(0) \mu \nu}=$ $-\Theta^{\alpha B} A_{(0) \alpha} V_{B} g_{(0) \mu \nu}$. Such correction generally break the space-time symmetry associated with the metric tensor $g_{(0) \mu \nu}$.

As before, the Seiberg-Witten map produces first order terms in the Abelian gauge fields. Here one gets some additional contributions to $A_{\mu}^{(1)}$

$$
\begin{aligned}
A_{\mu}^{(1)}= & A_{(0) \mu}-\frac{1}{2} \Theta^{\rho \sigma}\left(A_{(0) \rho}\left(\partial_{\sigma} A_{(0) \mu}+F_{(0) \sigma \mu}\right)\right. \\
& \left.+\frac{1}{8}\left[\omega_{(0) a b}\right]_{\rho}\left(\partial_{\sigma} \omega_{(0) \mu}^{a b}+R_{(0) \sigma \mu}^{a b}\right)\right)+\mathcal{O}\left(\Theta^{2}\right),
\end{aligned}
$$

where $F_{(0)}$ is the Abelian curvature for the solution. $A_{\mu}^{(2)}$ has the same form as in (4.3). There are now also first order correction to the spin connections

$$
\omega_{\mu}^{a b}=\omega_{(0) \mu}^{a b}-\frac{1}{2} \Theta^{\rho \sigma}\left(\omega_{(0) \rho}^{a b}\left(\partial_{\sigma} A_{(0) \mu}+F_{(0) \sigma \mu}\right)+A_{(0) \rho}\left(\partial_{\sigma} \omega_{(0) \mu}^{a b}+R_{(0) \sigma \mu}^{a b}\right)\right)+\mathcal{O}\left(\Theta^{2}\right) .
$$

This will result in nonvanishing torsion two forms (3.3) at first order.

2. $\hat{A}^{(2)}$ satisfies the noncommutative Maxwell equation. If (4.1) holds we have the exact noncommutative solution

$$
\hat{e}_{\mu}^{a}=e_{(0) \mu}^{a}, \quad \hat{\omega}_{\mu}^{a}=\omega_{(0) \mu}^{a}, \quad \hat{A}_{\mu}^{(2)}=A_{(0) \mu}, \quad A_{\mu}^{(1)}=\hat{f}_{\mu}^{a}=0 .
$$

Upon applying the Seiberg-Witten map back to the commutative theory, one gets

$$
\begin{aligned}
e_{\mu}^{a} & =e_{(0) \mu}^{a}-\frac{1}{4} \Theta^{\rho \sigma} \epsilon_{b c d}^{a} \omega_{(0) \rho}^{b c} A_{(0) \sigma} e_{(0) \mu}^{d}+\mathcal{O}\left(\Theta^{2}\right), \\
f_{\mu}^{a} & =\frac{1}{4} \Theta^{\rho \sigma} \epsilon_{b c d}^{a} \omega_{(0) \rho}^{b c}\left(\partial_{\sigma} e_{(0) \mu}^{d}+\frac{1}{2} \omega_{(0) \sigma}^{d g} e_{(0) g \mu}\right)+\mathcal{O}\left(\Theta^{2}\right),
\end{aligned}
$$


which now produces no first order correction to $g_{(0) \mu \nu}$. The corrections to the Abelian gauge potentials and spin connections in this case are given by

$$
\begin{aligned}
& A_{\mu}^{(1)}=-\frac{1}{2} \Theta^{\rho \sigma}\left(-A_{(0) \rho}\left(\partial_{\sigma} A_{(0) \mu}+F_{(0) \sigma \mu}\right)+\frac{1}{8}\left[\omega_{(0) a b}\right]_{\rho}\left(\partial_{\sigma} \omega_{(0) \mu}^{a b}+R_{(0) \sigma \mu}^{a b}\right)\right)+\mathcal{O}\left(\Theta^{2}\right), \\
& A_{\mu}^{(2)}=A_{(0) \mu}+\frac{1}{32} \Theta^{\rho \sigma} \epsilon_{a b c d} \omega_{(0) \rho}^{a b}\left(\partial_{\sigma} \omega_{(0) \mu}^{c d}+R_{(0) \sigma \mu}^{c d}\right)+\mathcal{O}\left(\Theta^{2}\right), \\
& \omega_{\mu}^{a b}=\omega_{(0) \mu}^{a b}-\frac{1}{4} \epsilon_{c d}^{a b} \Theta^{\rho \sigma}\left(\omega_{(0) \rho}^{c d}\left(\partial_{\sigma} A_{(0) \mu}+F_{(0) \sigma \mu}\right)+A_{(0) \rho}\left(\partial_{\sigma} \omega_{(0) \mu}^{c d}+R_{(0) \sigma \mu}^{c d}\right)\right)+\mathcal{O}\left(\Theta^{2}\right) .
\end{aligned}
$$

Of course, a final possibility is that the noncommutative Maxwell equation involves both $\hat{A}^{(1)}$ and $\hat{A}^{(2)}$, i.e., a linear combination of $\hat{A}^{(1)}$ and $\hat{A}^{(2)}$ satisfies the noncommutative Maxwell equations. The first order corrections to the commutative solution will then be a linear combination of those for the two cases, and would in general lead to first order corrections to $g_{(0) \mu \nu}$.

\section{Reissner-Nordström solution}

We now use the results of the previous section to obtain first order corrections to the ReissnerNordström metric tensor. The invariant interval for the Reissner-Nordström solution is

$$
\begin{aligned}
& d s_{(0)}^{2}=-\Delta(r) d t^{2}+\Delta(r)^{-1} d r^{2}+r^{2}\left(d \theta^{2}+\sin ^{2} \theta d \phi^{2}\right), \\
& \Delta(r)=1-\frac{2 G M}{r}+\frac{G Q^{2}}{r^{2}} .
\end{aligned}
$$

Along with the Coulomb gauge field

$$
F_{(0)}=\frac{Q}{r^{2}} d r \wedge d t
$$

(8.1) and (8.2) describe a black hole with charge $Q$ and mass $M$. The metric tensor gives rise to two horizons at $r=r_{ \pm}$,

$$
r_{ \pm}=G\left(M \pm \sqrt{M^{2}-\frac{Q^{2}}{G}}\right)
$$

Introducing coordinates $x_{i}, i=1,2,3$, spanning $R^{3}$, we can re-write the invariant measure to have metric components

$$
g_{(0) 00}=-\Delta(r), \quad g_{(0) i j}=\left(\Delta(r)^{-1}-1\right) \hat{x}_{i} \hat{x}_{j}+\delta_{i j}, \quad g_{(0) 0 i}=0 .
$$

As this is a static solution, the condition (4.1) is satisfied for the case of time-space noncommutativity (5.4). Following the discussion in Section 7, we can construct two exact solutions, (7.1) and (7.5), of the noncommutative Einstein-Maxwell equations. We take the former, since we want to obtain first order corrections to the metric tensor. For simplicity, choose only $\Theta^{03}$ nonzero and take $A_{(0)}=-\frac{Q}{r} d t$. Upon substituting into (7.2) we obtain the new invariant measure $d s^{2}=d s_{(0)}^{2}+d s_{(1)}^{2}$, where the first order correction is

$$
d s_{(1)}^{2}=-\frac{\Theta^{03} Q}{r}\left(\Delta^{\prime}(r) \cos \theta\left(d t^{2}+\Delta(r)^{-2} d r^{2}\right)+2\left(\Delta(r)^{-1}-1\right) \sin \theta d r d \theta\right)
$$

the prime denoting a derivative in $r$. Thus

$$
\begin{aligned}
d s^{2}= & -\Sigma(r, \theta) d t^{2}+\Sigma(r, \theta)^{-1} d r^{2}+r^{2}\left(d \theta^{2}+\sin ^{2} \theta d \phi^{2}\right) \\
& -\frac{2 \Theta^{03} Q}{r}\left(\Delta(r)^{-1}-1\right) \sin \theta d r d \theta+\mathcal{O}\left(\Theta^{2}\right),
\end{aligned}
$$


where

$$
\Sigma(r, \theta)=\Delta(r)+\frac{\Theta^{03} Q}{r} \Delta^{\prime}(r) \cos \theta .
$$

Rotation invariance is therefore broken at first order in $\Theta^{03}$. The two horizons (8.3) are shifted to $r=r_{ \pm}+\delta r_{ \pm}$, where $\delta r_{ \pm}$is $\Theta^{03}$-dependent

$$
\delta r_{ \pm}=-\frac{\Theta^{03} Q}{r_{ \pm}} \cos \theta+\mathcal{O}\left(\Theta^{2}\right) .
$$

The first order corrections to the metric tensor vanish in the $Q \rightarrow 0$ limit, consistent with the results obtained in Section 6 for the Schwarzschild black hole. Additional roots of $\Sigma(r, \theta)$ may also occur, and they appear to be nontrivial. Noncommutative corrections to black hole horizons and their effect on the Hawking temperature have been previously computed in [29, 30, 31, 32]. Here such effects will be first order $\Theta$.

Using (7.3), first order corrections will also result in the Abelian gauge fields ${ }^{5}$, as well as in the spin connections (7.4), and they generalize the results of Section 6 .

\section{Concluding remarks}

We have constructed exact solutions to noncommutative gravity and shown that they can generate Abelian gauge fields which are first order in the noncommutative scale. Conversely, Abelian gauge fields, if present in the solution, give rise to first order corrections to the metric tensor. In both cases, the effects are expected to break the space-time symmetries associated with the solution.

For the case of black holes, we found that both electrostatic and magnetostatic type fields are generated when $\Theta^{0 i} \neq 0$. They fall off as $1 / r^{6}$ and resemble higher multipole fields, with $\Theta^{0 i} r_{s}^{2}$ playing a role analogous to the multipole moment. Since $\Theta^{0 i}$ is constant in this theory, the moments of multiple black holes are aligned, which may lead to a peculiar signature for the detection of microscopic black holes.

We got extremely tiny electric-type fields at the current time for the case of the flat expanding universe. The fields grow when we evolve back in time. If we go back to the time of the radiationmatter equality, their strength is nowhere near that claimed needed for the primordial field to seed an amplification process which can produce the currently observed intergalactic magnetic fields. The energy scales of the fields remain well below the radiation energy even if we go back to the beginning of the radiation era. On the other hand, the electric fields could be significant during an exponential inflationary era. We plan on addressing their effect on inflationary models in the future. One possibility is that they reach the critical strength for the creation of charged particle pairs from the vacuum.

The system of [11] which was studied here contains two Abelian gauge fields. We have entertained the notion that one of them may correspond to ordinary electromagnetism. Although from (3.1), the infinitesimal gauge variations of $A^{(1)}$ and $A^{(2)}$ are identical in the commutative theory, $A^{(1)}$ is the natural candidate for the electromagnetic potential since it is associated with the compact direction in $G L(2, C)$. Here note that finite (commutative) $G L(2, C)$ gauge transformations are of the form

$$
\mathcal{A} \rightarrow \Omega \mathcal{A} \Omega^{-1}+i \Omega d \Omega^{-1}, \quad \mathcal{E} \rightarrow \Omega \mathcal{E} \Omega^{-1}, \quad \Omega=e^{i \Lambda},
$$

where $\Lambda=\frac{1}{2} \lambda^{a b} \sigma_{a b}+\alpha^{(1)} 11+i \alpha^{(2)} \gamma_{5}$ now denote finite parameters. Concerning $A^{(2)}$, it will couple to the axial vector current when including fermions. The possibility of generalizing the

\footnotetext{
${ }^{5}$ They will include contributions found in [3] in the absence of gravity.
} 
internal Abelian gauge symmetries of this theory to standard model symmetries is of further interest.

Other avenues of research involve examining alternatives to (5.3) and (5.4), which are associated with constant commutation relations for the comoving coordinates. As was remarked in Section 5 and in [15], one would get larger noncommutative effects at earlier times if one can instead implement constant commutation relations for the 'physical', rather than the comoving, spatial coordinates, as this would introduce extra factors of $a(t)^{-1}$. However, even after including such factors, the fields may still be too small to play a role as a primordial field which seeds an amplification process that can produce the currently observed intergalactic magnetic fields.

The electric fields which emerge from this gravity theory play a passive role and only appear as a result of the Seiberg-Witten map to the commutative theory. It is of interest to promote them to dynamical fields. They are then expected to contribute symmetry breaking terms to the stress-energy tensor ((5.2), for the example of the flat expanding universe) and generate a back reaction on the space-time geometry. Abelian gauge fields which are linear in $\Theta$ will lead to second order back reaction terms in the metric tensor.

\section{A Appendix}

Here we give expressions for the noncommutative gauge variations, the curvature and torsion and the Seiberg-Witten map in terms of the component one forms $\hat{\omega}^{a b}, \hat{A}^{(S)}, \hat{e}^{a}$ and $\hat{f}^{a}$. From (2.6), the noncommutative $G L(2, C)$ gauge variations can be written as

$$
\begin{aligned}
\delta_{\hat{\Lambda}} \hat{\omega}^{a b}= & d \hat{\lambda}^{a b}+\frac{1}{2}\left(\left\{\hat{\omega}^{a c}, \hat{\lambda}_{c}^{b}\right\}_{\star}-\left\{\hat{\omega}^{b c}, \hat{\lambda}_{c}{ }^{a}\right\}_{\star}\right)+i\left[\hat{\omega}^{a b}, \hat{\alpha}^{(1)}\right]_{\star}+i\left[\hat{A}^{(1)}, \hat{\lambda}^{a b}\right]_{\star} \\
& +\frac{i}{2} \epsilon^{a b c d}\left(\left[\hat{\omega}_{c d}, \hat{\alpha}^{(2)}\right]_{\star}+\left[\hat{A}^{(2)}, \hat{\lambda}_{c d}\right]_{\star}\right), \\
\delta_{\hat{\Lambda}} \hat{A}^{(1)}= & d \hat{\alpha}^{(1)}+i\left[\hat{A}^{(1)}, \hat{\alpha}^{(1)}\right]_{\star}-i\left[\hat{A}^{(2)}, \hat{\alpha}^{(2)}\right]_{\star}+\frac{i}{8}\left[\hat{\omega}^{a b}, \hat{\lambda}_{a b}\right]_{\star}, \\
\delta_{\hat{\Lambda}} \hat{A}^{(2)}= & d \hat{\alpha}^{(2)}+i\left[\hat{A}^{(1)}, \hat{\alpha}^{(2)}\right]_{\star}-i\left[\hat{A}^{(2)}, \hat{\alpha}^{(1)}\right]_{\star}-\frac{i}{16} \epsilon^{a b c d}\left[\hat{\omega}_{a b}, \hat{\lambda}_{c d}\right]_{\star}, \\
\delta_{\hat{\Lambda}} \hat{e}^{a}= & \frac{1}{2}\left\{\hat{e}^{b}, \hat{\lambda}_{b}{ }^{a}\right\}_{\star}-\frac{i}{4} \epsilon^{a b c d}\left[\hat{f}_{b}, \hat{\lambda}_{c d}\right]_{\star}+i\left[\hat{e}^{a}, \hat{\alpha}^{(1)}\right]_{\star}+\left\{\hat{f}^{a}, \hat{\alpha}^{(2)}\right\}_{\star}, \\
\delta_{\hat{\Lambda}} \hat{f}^{a}= & \frac{1}{2}\left\{\hat{f}^{b}, \hat{\lambda}_{b}{ }^{a}\right\}_{\star}-\frac{i}{4} \epsilon^{a b c d}\left[\hat{e}_{b}, \hat{\lambda}_{c d}\right]_{\star}+i\left[\hat{f}^{a}, \hat{\alpha}^{(1)}\right]_{\star}+\left\{\hat{e}^{a}, \hat{\alpha}^{(2)}\right\}_{\star} .
\end{aligned}
$$

The components $\hat{R}^{a b}, \hat{F}^{(S)}, \hat{T}^{a}$ and $\hat{U}^{a}$ of the noncommutative curvature and torsion two forms are given by

$$
\begin{aligned}
& \hat{R}^{a b}=d \hat{\omega}^{a b}+\frac{1}{2}\left[\hat{\omega}^{a c}, \hat{\omega}_{c}^{b}\right]_{\star}+i\left\{\hat{\omega}^{a b}, \hat{A}^{(1)}\right\}_{\star}+\frac{i}{2} \epsilon^{a b c d}\left\{\hat{\omega}_{c d}, \hat{A}^{(2)}\right\}_{\star}, \\
& \hat{F}^{(1)}=d \hat{A}^{(1)}+\frac{i}{8} \hat{\omega}^{a b}{ }^{\star} \hat{\omega}_{a b}+i \hat{A}^{(1)} \stackrel{\star}{\wedge} \hat{A}^{(1)}-i \hat{A}^{(2)} \stackrel{\star}{\wedge}^{(2)}, \\
& \hat{F}^{(2)}=d \hat{A}^{(2)}-\frac{i}{16} \epsilon_{a b c d} \hat{\omega}^{a b} \wedge^{\star} \hat{\omega}^{c d}+i\left\{\hat{A}^{(1)}, \hat{A}^{(2)}\right\}_{\star}, \\
& \hat{T}^{a}=d \hat{e}^{a}+\frac{1}{2}\left[\hat{\omega}^{a b}, \hat{e}_{b}\right]_{\star}-\frac{i}{2} \epsilon_{a b c d}\left\{\hat{\omega}_{b c}, \hat{f}_{c}\right\}_{\star}+i\left\{\hat{A}^{(1)}, \hat{e}^{a}\right\}_{\star}-\left[\hat{A}^{(2)}, \hat{f}^{a}\right]_{\star}, \\
& \hat{U}^{a}=d \hat{f}^{a}+\frac{1}{2}\left[\hat{\omega}^{a b}, \hat{f}_{b}\right]_{\star}-\frac{i}{2} \epsilon_{a b c d}\left\{\hat{\omega}_{b c}, \hat{e}_{c}\right\}_{\star}+i\left\{\hat{A}^{(1)}, \hat{f}^{a}\right\}_{\star}-\left[\hat{A}^{(2)}, \hat{e}^{a}\right]_{\star} .
\end{aligned}
$$

Up to first order, the Seiberg-Witten maps for the component one forms $\hat{\omega}^{a b}, A^{(S)}, \hat{e}^{a}$ and $\hat{f}^{a}$ are

$$
\begin{aligned}
\hat{\omega}_{\mu}^{a b}= & \omega_{\mu}^{a b}+\frac{1}{2} \Theta^{\rho \sigma}\left(\omega_{\rho}^{a b}\left(\partial_{\sigma} A_{\mu}^{(1)}+F_{\sigma \mu}^{(1)}\right)+A_{\rho}^{(1)}\left(\partial_{\sigma} \omega_{\mu}^{a b}+R_{\sigma \mu}^{a b}\right)\right. \\
& \left.+\frac{1}{2} \epsilon^{a b}{ }_{c d}^{c d}\left(\partial_{\sigma} A_{\mu}^{(2)}+F_{\sigma \mu}^{(2)}\right)+\frac{1}{2} \epsilon^{a b}{ }_{c d} A_{\rho}^{(2)}\left(\partial_{\sigma} \omega_{\mu}^{c d}+R_{\sigma \mu}^{c d}\right)\right), \\
\hat{A}_{\mu}^{(1)}= & A_{\mu}^{(1)}+\frac{1}{2} \Theta^{\rho \sigma}\left(A_{\rho}^{(1)}\left(\partial_{\sigma} A_{\mu}^{(1)}+F_{\sigma \mu}^{(1)}\right)-A_{\rho}^{(2)}\left(\partial_{\sigma} A_{\mu}^{(2)}+F_{\sigma \mu}^{(2)}\right)+\frac{1}{8}\left[\omega_{a b}\right]_{\rho}\left(\partial_{\sigma} \omega_{\mu}^{a b}+R_{\sigma \mu}^{a b}\right)\right), \\
\hat{A}_{\mu}^{(2)}= & A_{\mu}^{(2)}+\frac{1}{2} \Theta^{\rho \sigma}\left(A_{\rho}^{(1)}\left(\partial_{\sigma} A_{\mu}^{(2)}+F_{\sigma \mu}^{(2)}\right)+A_{\rho}^{(2)}\left(\partial_{\sigma} A_{\mu}^{(1)}+F_{\sigma \mu}^{(1)}\right)\right. \\
& \left.-\frac{1}{16} \epsilon_{a b c d} \omega_{\rho}^{a b}\left(\partial_{\sigma} \omega_{\mu}^{c d}+R_{\sigma \mu}^{c d}\right)\right),
\end{aligned}
$$




$$
\begin{aligned}
\hat{e}_{\mu}^{a}= & e_{\mu}^{a}+\Theta^{\rho \sigma} A_{\rho}^{(1)}\left(\partial_{\sigma} e_{\mu}^{a}+\frac{1}{2} \omega_{\sigma}^{a g} e_{g \mu}-A_{\sigma}^{(2)} f_{\mu}^{a}\right)-\frac{1}{4} \Theta^{\rho \sigma} \epsilon_{b c d}^{a} \omega_{\rho}^{b c}\left(\partial_{\sigma} f_{\mu}^{d}+\frac{1}{2} \omega_{\sigma}^{d g} f_{g \mu}-A_{\sigma}^{(2)} e_{\mu}^{d}\right), \\
\hat{f}_{\mu}^{a}= & f_{\mu}^{a}+\Theta^{\rho \sigma} A_{\rho}^{(1)}\left(\partial_{\sigma} f_{\mu}^{a}+\frac{1}{2} \omega_{\sigma}^{a g} f_{g \mu}-A_{\sigma}^{(2)} e_{\mu}^{a}\right) \\
& -\frac{1}{4} \Theta^{\rho \sigma} \epsilon_{b c d}^{a} \omega_{\rho}^{b c}\left(\partial_{\sigma} e_{\mu}^{d}+\frac{1}{2} \omega_{\sigma}^{d g} e_{g \mu}-A_{\sigma}^{(2)} f_{\mu}^{d}\right) .
\end{aligned}
$$

\section{Acknowledgements}

I am very grateful to P. Aschieri, S. Fabi, B. Harms and N. Okada for valuable discussions.

\section{References}

[1] Doplicher S., Fredenhagen K., Roberts J.E., Spacetime quantization induced by classical gravity, Phys. Lett. B 331 (1994), 39-44.

[2] Stern A., Noncommutative point sources, Phys. Rev. Lett. 100 (2008), 061601, 4 pages, arXiv:0709.3831.

[3] Stern A., Particlelike solutions to classical noncommutative gauge theory, Phys. Rev. D 78 (2008), 065006, 11 pages, arXiv:0804.3121.

[4] Pinzul A., Stern A., Noncommutative $\mathrm{AdS}^{3}$ with quantized cosmological constant, Classical Quantum Gravity 23 (2006), 1009-1021, hep-th/0511071.

[5] Chaichian M., Tureanu A., Zet G., Corrections to Schwarzschild solution in noncommutative gauge theory of gravity, Phys. Lett. B $66 \mathbf{6 0}$ (2008), 573-578, arXiv:0710.2075.

Chaichian M., Setare M.R., Tureanu A., Zet G., On black holes and cosmological constant in noncommutative gauge theory of gravity, J. High Energy Phys. 2008 (2008), no. 4, 064, 18 pages, arXiv:0711.4546.

[6] Fabi S., Harms B., Stern A., Noncommutative corrections to the Robertson-Walker metric, Phys. Rev. D 78 (2008), 065037, 7 pages, arXiv:0808.0943.

[7] Calmet X., Kobakhidze A., Second order noncommutative corrections to gravity, Phys. Rev. D 74 (2006), 047702, 3 pages, hep-th/0605275.

[8] Banerjee R., Mukherjee P., Samanta S., Lie algebraic noncommutative gravity, Phys. Rev. D 75 (2007), 125020, 7 pages, hep-th/0703128.

[9] Seiberg N., Witten E., String theory and noncommutative geometry, J. High Energy Phys. 1999 (1999), no. 9, 032, 93 pages, hep-th/9908142.

[10] Chamseddine A.H., Deforming Einstein's gravity, Phys. Lett. B 504 (2001), 33-37, hep-th/0009153.

[11] Chamseddine A.H., $S L(2, C)$ gravity with a complex vierbein and its noncommutative extension, Phys. Rev. D 69 (2004), 024015, 8 pages, hep-th/0309166.

[12] Stern A., Particle classification and dynamics in $G L(2, C)$ gravity, Phys. Rev. D 79 (2009), 105017, 16 pages, arXiv:0903.0882.

[13] Aschieri P., Blohmann C., Dimitrijevic M., Meyer F., Schupp P., Wess J., A gravity theory on noncommutative spaces, Classical Quantum Gravity 22 (2005), 3511-3532, hep-th/0504183.

[14] Schupp P., Solodukhin S., Exact black hole solutions in noncommutative gravity, arXiv:0906.2724.

[15] Ohl T., Schenkel A., Cosmological and black hole spacetimes in twisted noncommutative gravity, J. High Energy Phys. 2009 (2009), no. 10, 052, 12 pages, arXiv:0906.2730.

[16] Dolan B.P., Gupta K.S., Stern A., Noncommutative BTZ black hole and discrete time, Classical Quantum Gravity 24 (2007), 1647-1655, hep-th/0611233.

Dolan B.P., Gupta K.S., Stern A., Noncommutativity and quantum structure of spacetime, J. Phys. Conf. Ser. 174 (2009), 012023, 6 pages.

[17] Groenewold H.J., On the principles of elementary quantum mechanics, Physica 12 (1946), 405-460.

[18] Moyal J.E., Quantum mechanics as a statistical theory, Proc. Cambridge Philos. Soc. 45 (1949), 99-124.

[19] Utiyama R., Invariant theoretical interpretation of interaction, Phys. Rev. 101 (1956), 1597-1607.

[20] Kibble T.W.B., Lorentz invariance and the gravitational field, J. Math. Phys. 2 (1961), 212-221.

[21] Hehl F.W., Von Der Heyde P., Kerlick G.D., Nester J.M., General relativity with spin and torsion: foundations and prospects, Rev. Modern Phys. 48 (1976), 393-416.

[22] Chamseddine A.H., Applications of the gauge principle to gravitational interactions, Int. J. Geom. Methods Mod. Phys. 3 (2006), 149-176, hep-th/0511074. 
[23] Isham C.J., Salam A., Strathdee J.A., $S L_{6, C}$ gauge invariance of Einstein like Lagrangians, Lett. Nuovo Cimento 5 (1972), 969-972.

[24] Jurco B., Schraml S., Schupp P., Wess J., Enveloping algebra valued gauge transformations for non-abelian gauge groups on non-commutative spaces, Eur. Phys. J. C Part. Fields 17 (2000), 521-526, hep-th/0006246.

[25] Bonora L., Schnabl M., Sheikh-Jabbari M.M., Tomasiello A., Noncommutative $S O(n)$ and $S p(n)$ gauge theories, Nuclear Phys. B 589 (2000), 461-474, hep-th/0006091.

[26] Marculescu S., Ruiz Ruiz F., Seiberg-Witten maps for $S O(1,3)$ gauge invariance and deformations of gravity, Phys. Rev. D 79 (2009), 025004, 18 pages, arXiv:0808.2066.

[27] Davis A.-C., Lilley M., Törnkvist O., Relaxing the bounds on primordial magnetic seed fields, Phys. Rev. D 60 (1999), 021301, 5 pages, astro-ph/9904022.

[28] Mazumdar A., Sheikh-Jabbari M.M., Noncommutativity in space and primordial magnetic field, Phys. Rev. Lett. 87 (2001), 011301, 4 pages, hep-ph/0012363.

[29] Nasseri F., Schwarzschild black hole in noncommutative spaces, Gen. Relativity Gravitation 37 (2005), 2223-2226, hep-th/0508051.

[30] Nozari K., Fazlpour B., Reissner-Nordström black hole thermodynamics in noncommutative spaces, Acta Phys. Polon. B 39 (2008), 1363-1374.

[31] Banerjee R., Majhi B.R., Samanta S., Noncommutative black hole thermodynamics, Phys. Rev. D 77 (2008), 124035, 8 pages, arXiv:0801.3583.

[32] Banerjee R., Majhi B.R., Modak S.K., Noncommutative Schwarzschild black hole and area law, Classical Quantum Gravity 26 (2009), 085010, 11 pages, arXiv:0802.2176. 